\title{
Pretargeted radioimmunotherapy: clinically more efficient than conventional radioimmunotherapy?
}

\author{
Caroline Rousseau • Françoise Kraeber-Bodéré • \\ Jacques Barbet • Jean-François Chatal
}

Published online: 13 June 2013

(C) Springer-Verlag Berlin Heidelberg 2013

The clinical efficacy of radioimmunotherapy (RIT) has been clearly documented in the consolidation situation, such as first-line treatment of indolent non-Hodgkin lymphoma after induction chemotherapy [1] or after salvage resection of liver metastases from colon carcinoma [2], when tumour targets have a small, preferably microscopic, size. Efficacy has been much less evident for large tumours, more than $1-2 \mathrm{~cm}$ in diameter, especially for radioresistant tumours that need a higher absorbed dose and consequently a higher injected activity to be killed. The level of this injected activity is limited by the haematological toxicity following irradiation of bone marrow by the radioimmunoconjugate. One way to solve this problem is to limit the duration of such irradiation by decoupling the injection of the immunoconjugate and that of the radioactive effector which, due to its small size, can distribute rapidly so that irradiation of normal tissues is limited. This is the principle of pretargeted RIT (PRIT) which has been under development for more than 20 years.

Two main approaches have been preclinically and clinically tested: one uses the interaction of avidin (or streptavidin) and biotin, and the other the interaction of bispecific antibody (BsMAb) and hapten. In this issue of the European Journal of Nuclear Medicine and Molecular Imaging, van Rij et al. [3]

C. Rousseau $\cdot$ F. Kraeber-Bodéré

Nuclear Medicine Department, Comprehensive Cancer Center ICO

Gauducheau, Saint Herblain, France

F. Kraeber-Bodéré

Nuclear Medicine Department, University Hospital, Nantes, France

J. Barbet

Nantes-Angers Cancer Research Center, 8 quai Moncousu, 44007 Nantes cedex, France

J. Barbet $\cdot$ J.-F. Chatal $(\bowtie)$

Groupement d'Intérêt Public Arronax, 1, rue Aronnax,

44817 Saint-Herblain Cedex, France

e-mail: chatal@arronax-nantes.fr report on the second of these approaches. After two decades of multiple preclinical and clinical studies in several tumour types and using different antibodies and radionuclides, it is time to wonder whether PRIT is indeed better than conventional RIT in terms of toxicity and efficacy.

\section{Preclinical comparison}

It is quite clear that in preclinical studies which compared RIT and PRIT in three different tumour types using the same antibodies, PRIT was unambiguously superior to RIT with higher efficacy and lower or equivalent haematological toxicity. In a mouse model of LS174T human colorectal cancer ${ }^{131}$ I-RIT using intact IgG was compared to ${ }^{131}$ I-PRIT using the same anticarcinoembryonic antigen (CEA) antibody [4]. For PRIT a BsMAb/hapten molar ratio of $2: 1$ was used with an injected activity of $111 \mathrm{MBq}$ of ${ }^{131} \mathrm{I}$ as compared with $12 \mathrm{MBq}$ for RIT. The maximum tumour uptake was lower with PRIT $(8.5 \% \mathrm{ID} / \mathrm{g}$ at $1 \mathrm{~h})$ than with RIT $(33 \% \mathrm{ID} / \mathrm{g}$ at 2 days), but resulted in an equivalent tumour absorbed dose (102 Gy versus 95 Gy) and a lower blood absorbed dose (15 Gy versus 33 Gy). With PRIT, tumour growth delay was superior at 150 days as compared to 53 days with RIT with an equivalent haematological toxicity. Longer term monitoring of treated animals and post-mortem examination of tumours showed that with PRIT, $33 \%$ of the animals were cured, compared to none with RIT [5].

Using the same anti-CEA antibody, a similar comparison study was performed in a mouse model of TT human medullary thyroid carcinoma (MTC) [6, 7]. For PRIT, the BsMAb/hapten molar ratio of 2:1 was the same as in the previous study and the injected activity was $92.5 \mathrm{MBq}$ for both approaches. Tumour growth delay was 86 days with PRIT as compared to 65 days with RIT, with a substantially higher haematological toxicity with RIT (89\% decrease in white blood cell count, $66 \%$ decrease in platelet count) than with PRIT (34 \% decrease in white blood cell count, $39 \%$ decrease 
in and platelet count). Recently, a new bispecific trivalent antiCEA $\times$ anti-histamine-succinyl-glycine (HSG) antibody (TF2) was developed using Dock-and-Lock technology and used in the pretargeting of a bivalent HSG hapten referred to as IMP288 [8].

These new reagents allow the labelling of the di-HSG peptide with several radionuclides including ${ }^{177} \mathrm{Lu}$. In a study performed in a mouse model of LS174T human colorectal cancer, mice treated with PRIT using an anti-CEA antibody with a BsMAb/hapten molar ratio of 18:1 and an injected activity of $26 \mathrm{MBq}$ of ${ }^{177} \mathrm{Lu}$ showed a median survival of 45 days as compared to 13 days in untreated mice and a weak haematological toxicity [9]. In this study PRIT was not compared to RIT. Such a comparison has been performed by van Rij et al. [3]. In this study PRIT using the anti-TROP-2 TF12 and the ${ }^{177} \mathrm{Lu}$-IMP288 peptide was compared with RIT in a mouse model of PC3 prostate cancer. For PRIT a higher molar ratio than in the initial studies (25:1 versus 2:1) was used with an equivalent injected activity for PRIT and RIT (13.7 MBq versus $11.1 \mathrm{MBq}$ ). The efficacy of PRIT was slightly inferior to that of RIT with a median survival of 90 days as compared to more than 120 days, but the haematological toxicity of PRIT was much lower. Using the same injected activity, a $81 \%$ decrease in white blood cell count and a $61 \%$ decrease in platelet count were observed with RIT as compared with no toxicity with PRIT. An optimization study of this novel Dockand-Lock technology has been performed in a mouse model of LS174T human colon cancer [10]. As in previous studies, the maximum tumour uptake appeared lower with PRIT than with RIT but tumour-to-nontumour ratios and especially tumour-toblood ratios were much higher. It was shown that high tumour absorbed doses could be obtained with short-lived radionuclides such as alpha particle-emitting ${ }^{211} \mathrm{At}$, whereas repeated injections of long half-life beta-emitting radionuclides such as ${ }^{177} \mathrm{Lu}$ could be needed to get the same efficacy.

\section{Clinical comparison}

Clinical comparison of PRIT and RIT is much harder. Ideally this comparison should be done in a randomized trial using the maximum tolerated dose previously determined in phase I trials for each approach. In practice, such a comparison appears to be quite difficult if not impossible. Thus, the only way is to try to compare the efficacy and toxicity obtained in separate phases I/II trials using the same antibody and radionuclide. Such clinical studies have been performed in patients with metastatic MTC and colorectal carcinoma.

Medullary thyroid carcinoma

In one phase I/II trial and one phase II trial ${ }^{131}$ I-PRIT was assessed in, respectively, 26 and 42 patients with advanced
MTC $[11,12]$, and in one phase I/II trial ${ }^{131}$ I-RIT using the anti-CEA MN-14 antibody was assessed in 15 patients with MTC [13]. The pretherapeutic calcitonin serum levels were equivalent in the two phase I/II studies $(4,990$ and $5,900 \mathrm{pg} / \mathrm{ml}$ ) suggesting roughly equivalent advanced disease. Injected activities for RIT varied from $3.7 \mathrm{GBq}$ to $10 \mathrm{GBq}(99$ to $270 \mathrm{mCi}$ ) with 14 patients injected with more than $3.7 \mathrm{GBq}$ $(100 \mathrm{mCi})$ and for PRIT from $1.4 \mathrm{GBq}$ to $4.1 \mathrm{GBq}$ (38 to $112 \mathrm{mCi}$ ) with 4 patients injected with more than $3.7 \mathrm{GBq}$ $(100 \mathrm{mCi})$. Considering radiological and biological efficacy, stabilization was observed with RIT in 11 patients of 12 injected with a relatively high activity $(>5.2 \mathrm{GBq}$ or $140 \mathrm{mCi}$ ) and with PRIT in 6 patients of 17 including 3 of 4 injected with more than $3.7 \mathrm{GBq}(100 \mathrm{mCi})$. Haematological toxicity was slightly higher with RIT (grade $3 / 4$ in $47 \%$ of patients) than with PRIT (grade $3 / 4$ in $31 \%$ of patients). Based on these results, we consider that it is not possible to conclude that PRIT is superior to RIT in terms of efficacy or toxicity. Bone marrow involvement has been reported in $76 \%$ of patients with advanced MTC [14], and this could probably explain the high haematological toxicity, even with PRIT. The follow-up in patients in the PRIT study has been extended over a long period allowing the overall survival (OS) to be evaluated in comparison with that in 39 contemporaneous untreated patients with MTC with comparable prognostic indicators [15]. OS was significantly longer in the high-risk treated patients than in the high-risk untreated patients (median OS 110 and 61 months, respectively; $P<.030$ ). Unfortunately, this survival benefit of PRIT cannot be compared with that in the RIT study, in which the follow-up was much shorter. The phase II trial confirmed the efficacy of PRIT in progressive metastatic MTC, with a $76.2 \%$ disease control rate [12].

\section{Colorectal carcinoma}

In patients with metastatic colorectal cancer two phase I/II trials have been performed with ${ }^{131}$ I-RIT and ${ }^{131}$ I-PRIT using $\mathrm{MN}-14$ and F6 anti-CEA antibodies in, respectively, 17 and 8 patients $[16,17]$. The pretherapeutic CEA serum levels were equivalent in the two studies ( 73 and $77 \mathrm{ng} / \mathrm{ml}$ ) suggesting a roughly equivalent disease advancement. Injected activities with RIT varied from 2.1 GBq to 4.1 $\mathrm{GBq}(57.5$ to $110 \mathrm{mCi})$ with four patients injected with more than $3.7 \mathrm{GBq}(100 \mathrm{mCi})$ and with PRIT from $1.9 \mathrm{GBq}$ to $5.5 \mathrm{GBq}(51$ to $149 \mathrm{mCi}$ ) with three patients injected with more than $3.7 \mathrm{GBq}(100 \mathrm{mCi})$. No response was observed with RIT, whereas two patients injected with, respectively, 3 and $5.5 \mathrm{GBq}$ had stabilization with PRIT. Interestingly, three of seven patients injected with more than $3.7 \mathrm{GBq}$ had grade 4 haematological toxicity with RIT versus no patient with PRIT. These findings could mean that bone marrow involvement is less frequent in colorectal cancer than in MTC, and 
that it may then be possible to inject a higher activity with PRIT.

\section{Conclusion}

Although PRIT appears clearly superior to RIT in all preclinical studies, it is not currently possible to claim that PRIT is clinically superior to RIT. However, the optimization of PRIT continues with Dock-and-Lock technology paying particular attention to the BsMAb/hapten molar ratio, which must be high to optimize tumour uptake (20:1 or higher). The specific activity of the radionuclide then becomes important. Radionuclides with a shorter half-life, such as ${ }^{90} \mathrm{Y}$ and ${ }^{211} \mathrm{At}$, may prove more efficient than ${ }^{131} \mathrm{I}$ and the use of no carrier added ${ }^{177} \mathrm{Lu}$, instead of conventional ${ }^{177} \mathrm{Lu}$, is probably mandatory.

Regarding the most favourable clinical indication for documenting the potential advantage of PRIT, colorectal cancer could be relevant based on a previous phase II clinical study in which patients were injected with $2.2 \mathrm{GBq} / \mathrm{m}^{2}$ of ${ }^{131}$ I-labelled anti-CEA antibody. Only 1 of 28 patients had a grade 4 thrombocytopenia [18]. In the same clinical situation it would be interesting to check whether the optimized PRIT technique would allow the injected activity to be substantially increased for better efficacy without increasing haematological toxicity. Two phase I/II trials are ongoing in Europe using new-generation PRIT reagents in lung and colorectal carcinomas to optimize PRIT parameters.

In addition to therapeutic applications the pretargeting technique could be useful for immuno-PET applications. Imaging with intact antibodies requires a non-ideal delay of 4 to 7 days after injection before high-contrast images can be obtained. Imaging studies with smaller antibody fragments have demonstrated the possibility of obtaining images on the day of injection or the next day [19]. Pretargeting allows imaging within $1 \mathrm{~h}$ of injection of radiolabelled peptide with high contrast in animal models [20]. Two phase I/II trials are ongoing in France using new-generation pretargeted reagents in MTC and breast carcinomas to optimize pretargeted immuno-PET parameters.

\section{References}

1. Press OW, Palanca-Wessels MC. Selection of first-line therapy for advanced follicular lymphoma. J Clin Oncol. 2013;31:14969.

2. Liersch T, Meller J, Kulle B, Behr TM, Markus P, Langer C, et al. Phase II trial of carcinoembryonic antigen radioimmunotherapy with 131I-labetuzumab after salvage resection of colorectal metastases in the liver: five-year safety and efficacy results. J Clin Oncol. 2005;23:6763-70.
3. van Rij CM, Lütje S, Frielink C, Sharkey RM, Goldenberg DM, Franssen GM, et al. Pretargeted immuno-PET and radioimmunotherapy of prostate cancer with an anti-TROP-2 $\mathrm{x}$ anti-HSG bispecific antibody. Eur J Nucl Med Mol Imaging. 2013. doi:10.1007/ s00259-013-2434-7.

4. Gautherot E, Bouhou J, Le Doussal JM, Manetti C, Martin M, Rouvier E, et al. Therapy for colon carcinoma xenografts with bispecific antibody-targeted, iodine-131-labeled bivalent hapten. Cancer. 1997;80:2618-23.

5. Gautherot E, Rouvier E, Daniel L, Loucif E, Bouhou J, Manetti C, et al. Pretargeted radioimmunotherapy of human colorectal xenografts with bispecific antibody and 131I-labeled bivalent hapten. J Nucl Med. 2000;41:480-7.

6. Kraeber-Bodere F, Faivre-Chauvet A, Saï-Maurel C, Campion L, Fiche $\mathrm{M}$, Gautherot E, et al. Toxicity and efficacy of radioimmunotherapy in carcinoembryonic antigen-producing medullary thyroid cancer xenograft: comparison of iodine 131-labeled $\mathrm{F}(\mathrm{ab}$ ') 2 and pretargeted bivalent hapten and evaluation of repeated injections. Clin Cancer Res. $1999 ; 5: 3183 \mathrm{~s}-9 \mathrm{~s}$.

7. Kraeber-Bodere F, Faivre-Chauvet A, Saï-Maurel C, Gautherot E, Fiche M, Campion L, et al. Comparative toxicity and efficacy of one- and two-step (bispecific antibody and bivalent hapten) radioimmunotherapy in CEA-producing medullary thyroid cancer. J Nucl Med. 1999;40:198-204.

8. Rossi EA, Goldenberg DM, Cardillo TM, McBride WJ, Sharkey $\mathrm{RM}$, Chang CH. Stably tethered multifunctional structures of defined composition made by the dock and lock method for use in cancer targeting. Proc Natl Acad Sci U S A. 2006;103:6841-6.

9. Schoffelen R, van der Graaf WTA, Franssen G, Sharkey RM, Goldenberg DM, McBride WJ, et al. Pretargeted $177 \mathrm{Lu}$ radioimmunotherapy of carcinoembryonic antigen-expressing human colonic tumors in mice. J Nucl Med. 2010;51:1780-7.

10. Frampas E, Maurel C, Remaud-Le Saëc P, Mauxion T, FaivreChauvet A, Davodeau F, et al. Pretargeted radioimmunotherapy of colorectal cancer metastases: models and pharmacokinetics predict influence of the physical and radiochemical properties of the radionuclide. Eur J Nucl Med Mol Imaging. 2011;38:2153-64.

11. Kraeber-Bodéré F, Bardet S, Hoefnagel CA, Vieira MR, Vuillez JP, Murat A, et al. Radioimmunotherapy in medullary thyroid cancer using bispecific antibody and iodine 131-labeled bivalent hapten: preliminary results of a phase I/II clinical trial. Clin Cancer Res. 1999;5:3190s-8s.

12. Salaun PY, Campion L, Bournaud C, Faivre-Chauvet A, Vuillez JP, Taieb D, et al. Phase II trial of anticarcinoembryonic antigen pretargeted radioimmunotherapy in progressive metastatic medullary thyroid carcinoma: biomarker response and survival improvement. J Nucl Med. 2012;53:1185-92.

13. Juweid ME, Hajjar G, Swayne LC, Sharkey RM, Suleiman S, Herkovic T, et al. Phase I/II trial of 131I-MN-14 F(ab)2 anticarcinoembryonic antigen monoclonal antibody in the treatment of patients with metastatic medullary thyroid carcinoma. Cancer. 1999;85:1828-42.

14. Mirallié E, Vuillez JP, Bardet S, Frampas E, Dupas B, Ferrer L, et al. High frequency of bone/bone marrow involvement in advanced medullary thyroid cancer. J Clin Endocrinol Metab. 2005;90:779-88.

15. Chatal JF, Campion L, Kraeber-Bodere F, Bardet S, Vuillez JP, Charbonnel B, et al. Survival improvement in patients with medullary thyroid carcinoma who undergo pretargeted anti-carcinoembryonicantigen radioimmunotherapy: a collaborative study with the French Endocrine Tumor Group. J Clin Oncol. 2006;24:1705-11.

16. Hajjar G, Sharkey RM, Burton J, Zhang CH, Yeldell D, Matthies A, et al. Phase I radioimmunotherapy trial with iodine-131-labeled humanized $\mathrm{MN}-14$ anti-carcinoembryonic antigen monoclonal antibody in patients with metastatic gastrointestinal and colorectal cancer. Clin Colorectal Cancer. 2002;2:31-42. 
17. Kraeber-Bodere F, Rousseau C, Bodet-Milin C, Ferrer L, FaivreChauvet A, Campion L, et al. Targeting, toxicity, and efficacy of 2step, pretargeted radioimmunotherapy using a chimeric bispecific antibody and 131I-labeled bivalent hapten in a phase I optimization clinical trial. J Nucl Med. 2006;47:247-55.

18. Behr TM, Liersch T, Greiner-Bechert L, Griesinger F, Béhé M, Markus PM, et al. Radioimmunotherapy of small-volume disease of metastatic colorectal cancer. Cancer. 2002;94:1373-81.
19. Knowles SM, Wu AM. Advances in immuno-positron emission tomography: antibodies for molecular imaging in oncology. J Clin Oncol. 2012;30:3884-92.

20. Schoffelen R, van der Graaf WT, Sharkey RM, Franssen GM, McBride WJ, Chang $\mathrm{CH}$, et al. Pretargeted immuno-PET of CEA-expressing intraperitoneal human colonic tumor xenografts: a new sensitive detection method. EJNMMI Res. 2012;2:5. 